\title{
The Selection and Optimization of the Reconfigurable Shaped Reflector Structure Material
}

\author{
Hang Zhao ${ }^{1}$, Houfei Fang ${ }^{3}$, Matthew J. Santer ${ }^{2}$, Lan Lan ${ }^{3}$, Yangqing Hou $^{3}, \mathrm{Ke} \mathrm{Wu}^{1}$, Hengkun Jiang ${ }^{1}$ \\ ${ }^{1}$ China Academy of Space Technology, 710000 Xi'an, China \\ ${ }^{2}$ Department of Aeronautics, Imperial College, SW7 2AZ London, UK \\ ${ }^{3}$ Shanghai YS Information Technology Co.,Ltd, 200240 Shanghai, China
}

\begin{abstract}
The method of applying deformable 2-D lattice materials in design of the main structure of a reconfigurable shaped reflector is proposed in this paper. Hex-chiral NPR (Negative Poisson's Ratio) lattice, re-entrant NPR lattice and star ZPR (Zero Poisson's Ratio) lattice are investigated in forming the main structure of a reflector, according to the mechanical properties requirement. Finite element models of reflectors built by these three types of lattice materials are developed. An example of a reflector with reconfigurable shape, which is transformed from a standard paraboloid, is given. The curvature change of the deformed shape is calculated. Then, the region with the largest curvature changing rate is found and the configuration of such area is regenerated. Finally, the surface accuracy of the region with the largest curvature changing rate is evaluated for reflectors built by the three compared lattice materials. The simulation results show that the highest surface accuracy is obtained when applying the hex-chiral lattice to the design of the reconfigurable shaped reflector.
\end{abstract}

\section{Introduction}

In recent years, due to the development and demand of satellite communication, on-orbit reconfigurable antenna has experienced great research interest and practical value. Among the methods of on-orbit reconfiguration, the mechanical reconstruction of the reflective surface is a viable and economical approach [1-3]. For a mechanical reconfigurable shaped reflector, the surface of the shaped reflector material is deformed to a specific shape, so as to meet the requirements of the radiation field. For such reason, special demands are placed on the reflector surface material in order to meet the basic material strength and electrical performance requirements and to have a certain degree of elastic deformation.

The work on reconfigurable antennas was performed by many researchers. Leri et al.[4] applied a flexible composite material to form the reflecting surface structure and performed reconfigurable experiments. The structure has higher reconstruction shaping accuracy as well as generates a local buckling due to the stress concentration; Song S Y et al.[5] reconstructed the planar reflector structure of carbon fiber honeycomb sandwich structure with displacement of several millimeters; Lanlan et al.[6] studied the active surface control of high-precision solid surface reflector under piezoelectric actuators, which renders high surface control accuracy, yet structural deformation only on the order of microns.

So far, it is essential to have a type of applicable and efficiently shaped reflective surface structure material which can achieve a large deformation in the elastic range for the mechanical reconfigurable antenna field. Negative Poisson's Ratio (NPR) and Zero Poisson's Ratio (ZPR) lattice materials have the potential to solve this critical problem by virtue of the in-cell deformation of the material that produces hyperbolic dome-shaped or singlecurvature arched surfaces under out-of-plane loads. This avoids excessive stress, such as hex-chiral NPR lattice material[7], re-entrant hexagonal NPR lattice material[8], star ZPR lattice material[9].

In the fields of industry and medicine, researchers have used the re-entrant hexagonal NPR lattice materials to manufacture deformable functional structures such as chairs[10], filters[11] and medical bandages[12], and have achieved good results.

In the field of aerospace, Spodini[13] used a new NPR lattice material as the structural material of aircraft wings to accomplish wing deformation along the wing rib direction. Micheal et al.[14] put a similar NPR lattice material into the study of the out-of-plane deformation of the wing, which serves as a vortex generator to change the flow characteristics.

To accommodate the requirements of mechanical properties and the size parameters of lattice material cells to optimize the design space, this paper investigates a variety of NPR and ZPR lattice materials and utilizes hexchiral NPR lattice, re-entrant hexagonal NPR lattice and star ZPR lattice material as the structural material of the reconfigurable shaped reflector surfaces. To validate and compare the feasibility and reconstruction shaped accuracy of the three types of lattices as reconfigurable shaped reflector, an example is selected from the initial

\footnotetext{
* Corresponding author: nuanhuo17@126.com
} 
standard paraboloid to apply load through actuators to reconstruct deformation as the target shaped reflector surface. The RMS error between the deformed reflector surface and the target shaped reflection surface is tested to determine whether the shaped reflection surface with the lattice material as the basic structure meets the requirements. Meanwhile, the reflector surfaces of the three materials are impacted in the same local area by actuators in the finite element simulation. Through comparing the highest precision that the three can achieve, the forming accuracy of the hex-chiral NPR lattice material is found to be the highest.

\section{The selection of lattice material}

After investigating a variety of two-dimensional lattice materials, the hex-chiral NPR lattice, the re-entrant hexagonal NPR lattice material and the star ZPR shown in Fig. 1 are selected as the structural materials of the reconfigurable shaped reflector surface respectively. The reasons are as follows: 1) The Poisson's ratio of hex-chiral lattice material remains almost unchanged within a certain strain range, and the lattice property is quasi-isotropic[7]; 2) Re-entrant hexagon NPR lattice has large deformation capacity[8]. As being well studied recently, it was proven to be a strong candidate in forming reconfigurable shaped reflector surface. 3) In addition, the star ZPR lattice, which is a zero Poisson's ratio lattice material, was selected for deep analysis and comparison. Under uniaxial tensions, the length of the material in the direction orthogonal to the tensions almost remains unchanged. Therefore, this material can be used to generate singlecurvature out-of-plane deformation; 4) The cell size parameters of these three kinds of lattice materials are numerous. Thus, they offer more design freedom for subsequent optimization of the dimensional parameters.
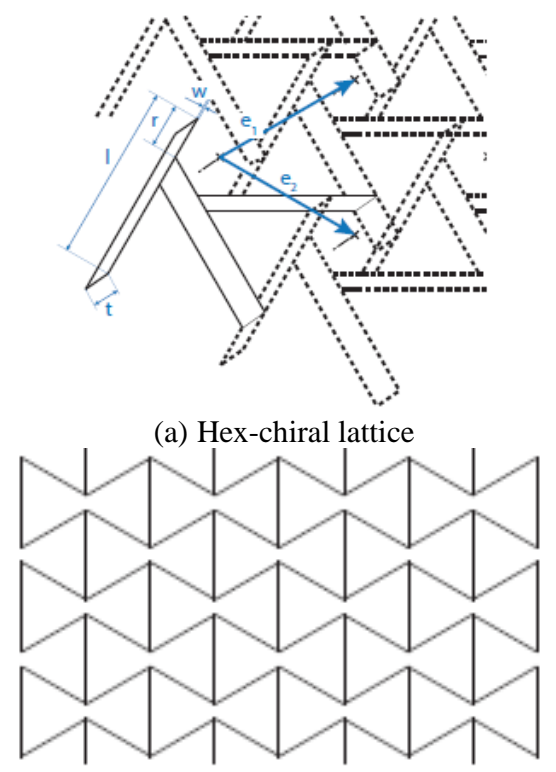

(b) Re-entrant lattice

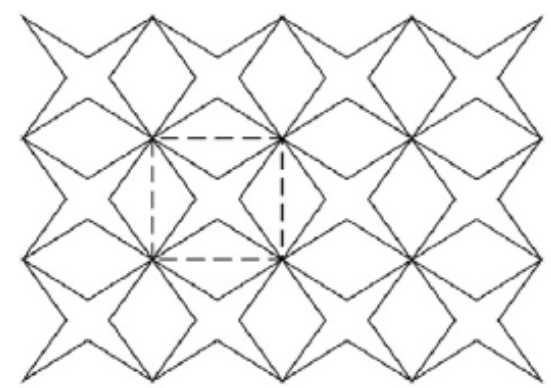

(c) Star lattice

Figure 1. The unit cell topology of the three types of lattice material

\section{Calculation of Deformation Curvature of the Reconfigurable Shaped Reflector Surface}

According to the required frequency band of the reflector the cell size of the lattice material constituting the reflector main body structure ranges from $5 \mathrm{~mm}$ to $75 \mathrm{~mm}$.

The actuators are required for the reflector to transfer from initial surface to target surface. If the local area with the largest change in Gaussian curvature can be changed by a certain number of actuators to meet the RMS accuracy requirement, the shaped deformation of the entire reflection surface is considered satisfied. Therefore, to compare the forming ability of the three lattice materials, an example is given in this paper in which the abilities of the three types of lattice materials to deform are compared by transferring a standard parabolic reflective surface into a target shaped reflective surface and by the RMS errors.

The method of calculating the principal curvature and the Gaussian curvature[15] is as follows: First, the local surface fitting formula of the local area is fitted by the local best fitting method of fitting the nine points, including the center point and its peripheral points:

$$
F(x, y, z)=a x^{2}+b y^{2}+c z^{2}+e x y+f y z+g z x+l x+m y+n z
$$

The coefficient terms in the first and second basic forms of the equation are obtained by the differential geometry:

$$
\begin{aligned}
& E=\left(1^{2}+n^{2}\right) / n^{2}, F=l m / n^{2}, G=\left(m^{2}+n^{2}\right) / n^{2} \\
& L=\frac{2\left(g n l-c l^{2}-a n^{2}\right)}{n^{2} \sqrt{l^{2}+m^{2}+n^{2}}} \\
& M=\frac{f n l+g m n-e n^{2}-2 c l m}{n^{2} \sqrt{l^{2}+m^{2}+n^{2}}} \\
& N=\frac{2\left(f m n-c m^{2}-b n^{2}\right)}{n^{2} \sqrt{l^{2}+m^{2}+n^{2}}}
\end{aligned}
$$

Second, the principal curvature and the Gaussian curvature can be obtained by the following formula:

$$
\left\{\begin{array}{c}
k_{1}=\frac{2 M F-L G-N E+\sqrt{(N E-L G)^{2}+4(L F-M E)(N F-M G)}}{2\left(E G-F^{2}\right)} \\
k_{2}=\frac{2 M F-L G-N E-\sqrt{(N E-L G)^{2}+4(L F-M E)(N F-M G)}}{2\left(E G-F^{2}\right)} \\
k_{g}=\frac{L N-M^{2}}{E G-F^{2}}
\end{array}\right.
$$


By calculating the above, the maximum principal curvature of the forming surface on the reflective surface is found to be $1.26 \mathrm{~m}^{-1}$ and the maximum Gaussian curvature is $0.5 \mathrm{~m}^{-2}$.

The Gaussian curvature variation of the reflecting surface from the standard surface to the target forming surface is shown in Fig. 2. The local area where the Gaussian curvature changes the most is shaped by applying seven displacement actuators as shown in the figure.

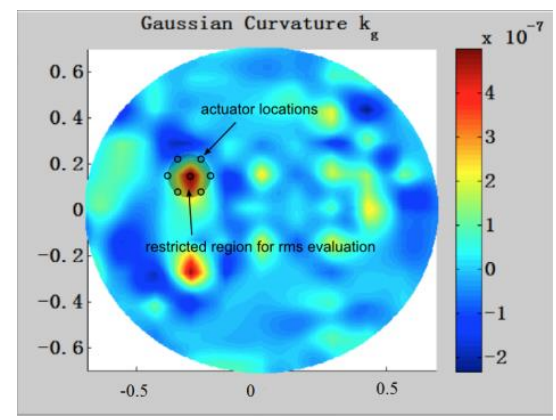

Figure 2. The cloud map of the Gaussian curvature of the shaped deformation and schematic diagram of the loaded actuators of the local area

\section{Finite element modeling}

Based on the above analysis, to determine how to establish the finite element model, as shown in Fig. 3, the components of the reconfigurable shaped reflector must include a reflective surface and seven actuators arranged in the middle of said surface. The reflective surfaces are made of lattice materials. Each of the three different lattice materials are compared through modeling, and the appropriate material is determined from the comparison.

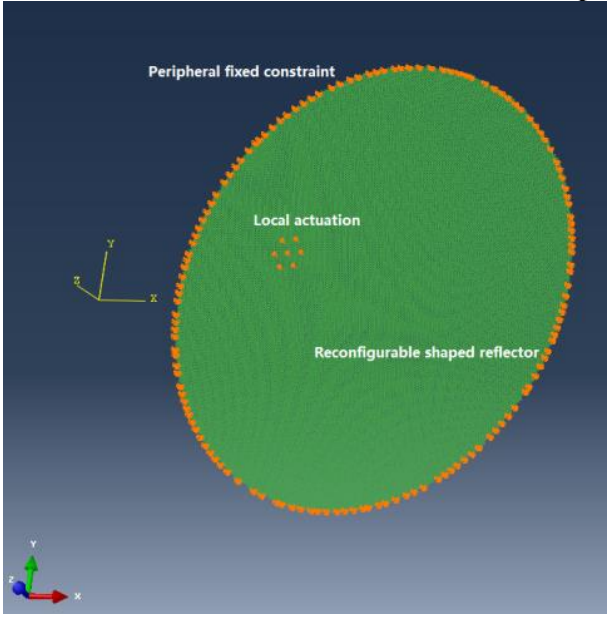

Figure 3. A schematic diagram of the constituent parts of the reconfigurable reflecting surface

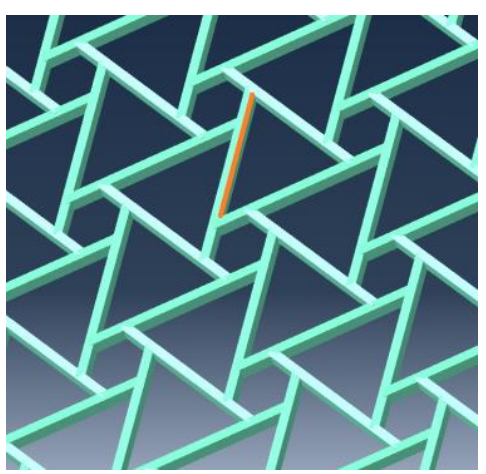

(a) Hex-chiral lattice

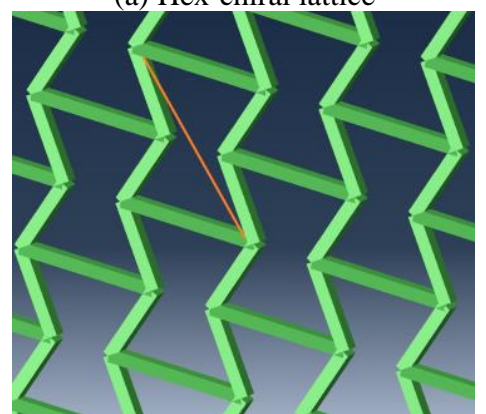

(b) Re-entrant lattice

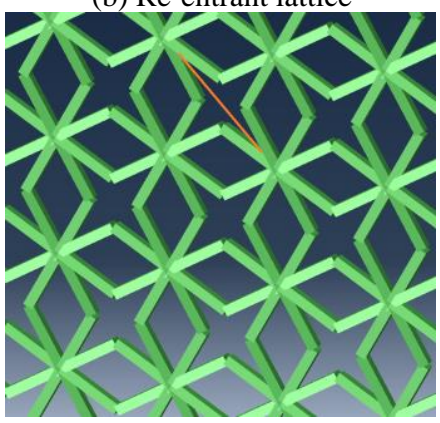

(c) ZPR-Star lattice

Figure 4. Three kinds of lattice material cell enlargement and the largest hole which wave could get through the pores

(orange line)

To ensure same preconditions, the maximum pore, wall thickness, and wall height of the three kinds of lattice materials are kept the same and the same forming area is selected. The maximum cell aperture is $8 \mathrm{~mm}$, the wall thickness (w in Fig. 1a) is 1mm, the wall height ( $t$ in Fig. 1a) is $2 \mathrm{~mm}$, and the paraboloid structures of the corresponding size lattice materials are established accordingly. Local cell enlargements are shown in Fig. 4.

Because the ratio of cell wall thickness and wall height to cell length is less than $1 / 10$, the element in the reflection surface model of ABAQUS finite element software selected beam element B31. This element type allows shear deformation and takes into account the limited axial strain, so it is suitable both for simulating slender beams and also for simulating stout beams. Since the reflector body is deformable and the actuator stiffness is relatively large, the actuators used are discrete rigid body units.

Considering the external ambient temperature, structural rigidity, and reconstruction toughness requirements of the reflector when working in orbit, titanium alloy should used as the matrix material of the lattice material, which has the advantage of high strength, good corrosion resistance, and excellent high/low 
temperature performance. The elastic modulus of titanium is $113 \mathrm{GPa}$; the Poisson's ratio is 0.3 ; the density is 4500 $\mathrm{kg} / \mathrm{m}^{3}$; and the yield strength is $1.05 \mathrm{GPa}$.

The constraints of the model analysis are as follows: fixed constraints are imposed on the circumferential edges of the reflecting surface; displacement loads are applied by seven local actuators, and the displacement load is determined according to the optimization method in the next section.

\section{Deformation ability analysis}

RMS error defined as the difference between reflecting surface and the target formation surface, is used to evaluate the shaping ability of the reflective surface of the lattice material. A smaller RMS error indicates a reflecting surface closer to the target formation surface.

RMS error can be calculated in various ways [16]. In this paper, the theoretical formula for calculating RMS error used is:

$$
R M S=\sqrt{\frac{\sum_{i=1}^{n}\left(X_{i}-R_{i}\right)^{2}}{n}}
$$

Where $X_{i}$ is the out-of-plane displacement of the $i$ node, and $\mathrm{R}_{\mathrm{i}}$ is the target displacement of the $\mathrm{i}$ node, $\mathrm{n}$ is the total number of all the nodes in the circular local region involved in the calculation of RMS (in which the local area of the star lattice has 4658 nodes, the re-entrant hexagon has 5010 nodes, the hex-chiral has 4962 nodes).

To optimize the RMS error between the actuatorloaded profile and the target profile, the load displacements of the actuators need to be optimized by numerical algorithms [17]. The algorithm used is the gradient algorithm, so the face optimization problem can be expressed mathematically, as follows:

$$
\left\{\begin{array}{c}
\min \left\|\left\{\delta^{I}\right\}+\{\nabla \delta\}-\left\{\delta^{D}\right)\right\|_{2}^{2} \\
\text { s.t. }[K]\{x\}=\{\nabla \delta\} \\
\max \{x\} \leq\{x\}_{\max }
\end{array}\right.
$$

Where $\left\{\delta^{\mathrm{I}}\right\}$ is the initial position coordinates of the nodes in the local region involved in the calculation of the reflector, $\left\{\delta^{\mathrm{D}}\right\}$ is the target position of these nodes, $\{\nabla \delta\}$ is the displacement of the nodes, $\{\mathrm{x}\}$ is the loading displacement of the seven actuators, $\{x\}_{\max }$ is the maximum load displacement. $[\mathrm{K}]$ is the nodes' influence coefficient matrix which represents the influence of the actuator's displacement loads on the nodes' deformation displacement in the local area. For small deformation analysis, displacement change of all the nodes in the domain can be obtained by multiplying the matrix by the displacement vector of the 7 actuators. The optimization curves of the RMS errors for the reflecting surfaces of the three types of lattice materials are shown in Fig 5. They are constructed under the same conditions of convergence, with optimization of the magnitude of the actuators' loading displacement.

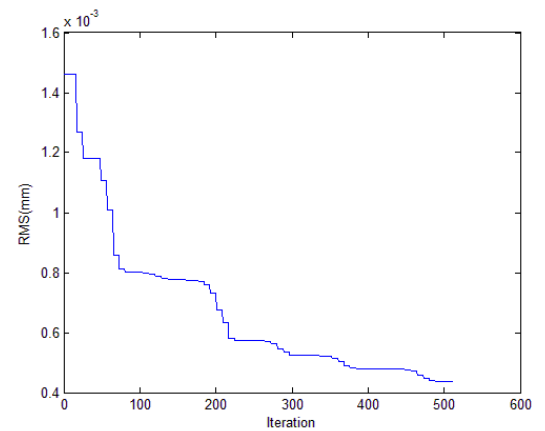

(a) The re-entrant hexagon lattice reflector

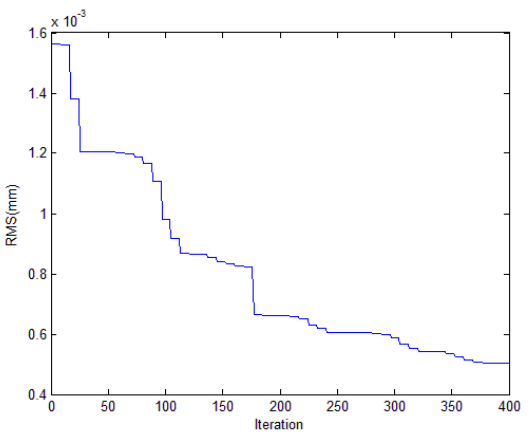

(b) The star lattice reflector

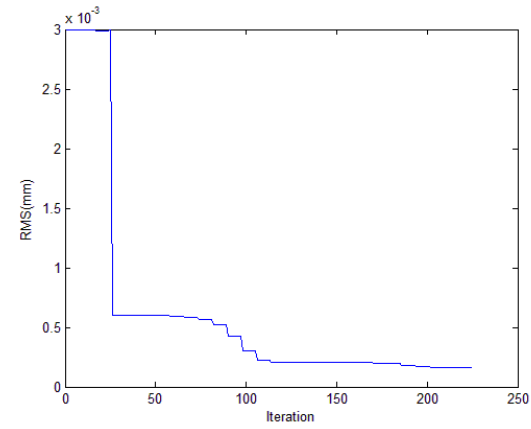

(c) The hex-chiral lattice reflector

Figure 5. The optimization curve of the RMS of the local area of the shaped reflector made of three lattice respectively

The optimized minimum RMS error of the shaping areas of the three kinds of lattice materials are shown in Table 1. The minimum RMS error of the re-entrant hexagonal lattice material and the star lattice material are close to each other, but both of them are much larger than the minimum RMS error of the reflective surface of the hex-chiral lattice material. This is because the deformation mechanism of the re-entrant hexagonal lattice material and the star lattice material is based on the "folding" of the rod inside the cell[8], its equivalent Poisson's ratio changes with the increase of the deformation so it becomes unstable. The deformation mechanism of the hex-chiral lattice material is based on the "rotation" effect of the hexagon inside the cell and the "bending" effect of the triangle[7]. Thus the equivalent Poisson's ratio is not increasing with the deformation change, and the material property is quasi-isotropic. Therefore the mechanical properties of the material is almost the same in all directions.

Based on the above analysis, the hex-chiral lattice material, as the basic structure of reconfigurable shaped reflection surface, renders good forming accuracy and meets the requirements of the application on the 
fundamental structure of reconfigurable shaped reflection surface.

Table 1. The comparison of the shaping ability of the reflection surface made of the three kinds of lattice material.

\begin{tabular}{|c|c|c|c|}
\hline $\begin{array}{c}\text { Lattice } \\
\text { material }\end{array}$ & $\begin{array}{c}\text { Re- } \\
\text { entrant }\end{array}$ & Star & Hex-chiral \\
\hline $\operatorname{RMS}(\mu \mathrm{m})$ & 438 & 501 & 155 \\
\hline
\end{tabular}

\section{Conclusion}

The requirements of the application for the mechanical properties of the materials were analysed by investigating the materials of reconfigurable shaped reflective surfaces. The materials for deformable functional structures in other areas including aerospace, and the medical field were investigated to explore the possibility of lattice materials as reconfigurable matrix substrate material.

Through the investigation of a variety of negative Poisson's ratio and zero Poisson's ratio lattice materials, with the design requirements to have the same local area with the largest change of curvature on the reflective surface, and the optimized displacement of the actuators of the three reflectors under the same convergence constraints, three kinds of lattice materials are found to be more suitable as the fundamental structure of the reflective surface.

The RMS errors, which measure the deviation between the initial reflective surface and the target surface, are compared. The hex-chiral lattice material, when serving as the fundamental structure of the reflector, renders significantly higher surface accuracy than the other two kinds of materials analyzed.

\section{References}

1. Washington G,Yoon $\mathrm{H}$ S,Angelino M,et al. Design,modeling,and optimization of mechanically reconfigurable aperture antennas[J]. IEEE Transactions on Antennas \& Propagation,2002,50(5):628-637.

2. Shi H, Yuan S, Yang B. New Methodology of Surface Mesh Geometry Design for Deployable Mesh Reflectors[J]. Journal of Spacecraft \& Rockets, 2017(9):1-16.

3. Yuan S, Yang B, \& Fang H. Form-Finding of Large Deployable Mesh Reflectors with Elastic Deformations of Supporting Structures. In 2018 AIAA Spacecraft Structures Conference (p. 1198).

4. Leri Datashvili, Horst Baier, Bin Wei and Stephan Endler. Design of a Morphing Skin Using Flexible Fiber Composites for Space-Reconfigurable Reflectors $[\mathrm{C}] / /$ Structures, Structural Dynamics, and Materials and Co-located Conferences. Boston: AIAA, 2013.
5. Song S.Y., Xu M.L.,Shao SH.B.. Structural Design of Mechanically Reconfigurable Reflector Antennas[J]. Space Electronic Technology,2016, 13(2):49-53.

6. Lan L, Jiang S, Zhou Y, et al. Shape Control of a Reflector Based on Generalized Zernike Functions[C]// AIAA Spacecraft Structures Conference. 2015.Prall D, Lakes R S. Properties of a chiral honeycomb with a poisson's ratio of - $1[\mathrm{~J}]$. International Journal of Mechanical Sciences, 1997 , 39(3):305-307.

7. Prall D, Lakes R S. Properties of a chiral honeycomb with a poisson's ratio of $-1[\mathrm{~J}]$. International Journal of Mechanical Sciences, 1997, 39(3):305-307.

8. Gonella S, Ruzzene M. Homogenization and Equivalent In-Plane Properties of Hexagonal and ReEntrant Honeycombs[C]// ASME 2007 International Mechanical Engineering Congress and Exposition. 2007:1117-1127.

9. Gong X, Huang J, Scarpa F, et al. Zero Poisson's ratio cellular structure for two-dimensional morphing applications[J]. Composite Structures, 2015, 134:384-392.

10. Du Y.T.. Study of Mechanical Properties of Morphing Honeycomb Structures Based on Shape Memory Polymer[D]. Harbin Institute of Technology, 2016.

11. Carneiro V H, Meireles J, Puga H. Auxetic materials - A review[J]. Materials Science-Poland, 2013, 31(4):561-571.

12. Mir M, Ali M, Ansari U, et al. Review of Mechanics and Applications of Auxetic Structures[J]. Advances in Materials Science \& Engineering, 2014, 2014.

13. Spadoni A. Application of chiral cellular materials for the design of innovative components[J]. Dissertations \& Theses - Gradworks, 2008.

14. M Garland, M Santer, J Morrison. Optimal AeroStructural Design of an Adaptive Surface for Boundary Layer Motivation Using an Auxetic Lattice Skin[J]. Journal of Intelligent Material Systems and Structures,2017.

15. Meek, D. S, Walton, et al. On surface normal and Gaussian curvature approximations given data sampled from a smooth surface[J]. Computer Aided Geometric Design, 2000, 17(6):521-543.

16. Yuan S, Yang B. Improvement of Surface Accuracy for Large Deployable Mesh Reflectors[C]// AIAA/AAS Astrodynamics Specialist Conference. 2016.

17. Yuan S, Yang B. Design and Optimization of Tension Distribution for Space Deployable Mesh Reflectors[C]// Aas/aiaa Space Flight Mechanics Meeting. 2016. 